\title{
Immunology and morphology studies on the proliferation of in vitro cultivated Echinococcus multilocularis metacestodes
}

Received: 13 January 1995 / Accepted: 23 March 1995

\begin{abstract}
The larval stage of Echinococcus multilocularis causes alveolar echinococcosis (AE) in various mammals, including humans. Traditionally metacestodes are maintained in the laboratory by serial transplantation passages into susceptible animals such as mice or gerbils. However, in animal models it has always been difficult to draw definite conclusions about the factors modulating metacestode differentiation, and investigations on gene expression and respective regulation have been hampered by the complexicity of the host-parasite interplay. This paper describes the maintenance and proliferation of E. multilocularis metacestodes as well as the formation of protoscolices in a chemically defined medium devoid of host influence. The interactive role of a heterologous human cell line (CACO2) in the in vitro development of metacestodes was also assessed. The morphology and ultrastructure of in vitro-generated metacestodes was studied using scanning (SEM) and transmission electron microscopy (TEM).Different cultivation procedures were analyzed in terms of expression of Band T-cell epitopes and of the relevant laminated layerantigen Em2; the exact localization of this antigen was further demonstrated by immunogold electron microscopy.
\end{abstract}

\section{Introduction}

In vitro cultivation and proliferation of the metacestode stage of Echinococcus multilocularis, the causative agent of alveolar echinococcosis (AE) in humans, was demonstrated by Rausch and Jentoft as early as in 1957, although the respective secondary vesicles may be devoid of the laminated layer around the cysts (Rausch and

\footnotetext{
A. Hemphill · B. Gottstein (

Institute of Parasitology,

Faculty of Veterinary Medicine and Faculty of Medicine,

University of Berne, Länggass-Strasse 122,

CH-3001 Bern, Switzerland

Fax: \#41 3163126 22,e-mail: gottstein@ipa.unibe.ch
}

Jentoft 1957) or even lack protoscolex formation within the cysts (Yamashita et al. 1962). However, the use of chemically non-defined media makes it difficult to investigate specifically the factors modulating parasite differentiation, and it becomes impossible to use the systems for molecular approaches to study gene expression and regulation during metacestode cell differentiation and proliferation.

Under defined in vitro culture conditions for E. multilocularis oncospheres, however, it has previously been shown that in vitro postoncospheral development is accompanied by the formation of a laminated layer starting within 13 days of culture (Deplazes and Gottstein 1991). This laminated layer contains the Em2 antigen, a dominant antigenic substance with lectin-binding properties (Gottstein 1985), which seems to play an important role in metacestode survival mechanisms within the immunologically reacting host (Gottstein et al. 1994), even with respect to the developing oncosphere (Sakamoto and Sugimura 1970). It has also been shown experimentally that only those E. multilocularis metacestode structures that have been capable of synthesizing an Em2-positive laminated layer exhibit the potential to induce secondary AE in rodents (Gottstein et al. 1992). Presently, little is known about the molecular mechanisms regulating metacestode differentiation, especially about the molecular synthesis of the laminated layer. These regulatory mechanisms can be elucidated in a biological system only independently of host influence. Thus, there is an urgent need for defined in vitro cultivation systems of the larval parasite. In this paper, we describe the in vitro maintenance and proliferation of cloned E. multilocularis metacestodes in a chemically defined medium, the modulation of protoscolex formation by mammalian host cell components, and the analysis of antigenic and morphological properties in dependence on cultivation conditions. The presented models may provide efficient tools for further molecular studies on the metacestode biology with special emphasis on in vitro-reconstituted host-parasite interactions. 


\section{Materials and methods}

\section{Experimental design}

To date, Echinococcus multilocularis has usually been maintained in the laboratory by serial transplantation passages of the metacestode into susceptible animals such as mice or gerbils. To establish an alternative host-free method for maintenance of the parasite, we elaborated an in vitro cultivation technique allowing the maintenance of metacestode strains under proliferation for an extended period. Cultivation included basically the following steps:

1. Metacestode tissue was aseptically removed from the peritoneal cavity of infected mice.

2. From step 1 , small tissue blocks $\left(0.5 \mathrm{~cm}^{3}\right)$ were placed into a suitable medium.

3. Alternatively, a vesicle suspension was prepared from step 1 .

4. Cultivation of tissue blocks and vesicles was carried out at $37^{\circ} \mathrm{C}$ under semi-anaerobic conditions. Follow-up parameters recorded during cultivation were the production, growth, and proliferation of secondary vesicles as well as the development of protoscolices within vesicles.

5. For assessment of growth promotion by heterologous host cells, a defined number of parasite vesicles was cultured in the presence or absence of $\mathrm{CACO} 2$ cells. Follow-up parameters again included the production, growth, and proliferation of secondary vesicles as well as protoscolex development. Additional end-point parameters used to assess different cultivation procedures comparatively included analysis of the antigenic profiles of different metacestode fractions (vesicle fluids, vesicle extracts, and medium supernatants). Antigen profile analysis was done with respect to (a) B-cell epitope expression, using immunoblotting and sera from patients with confirmed AE or cystic hydatid disease; (b) T-cell epitope expression, by testing lymphocytes of a patient suffering from $\mathrm{AE}$ with vesicle fluids, parasite extracts, and medium supernatants for the stimulation of an in vitro lymphoproliferative response; and (c) Em2-antigen expression. The presence of the Em2 antigen was monitored by testing the different parasite fractions with a double sandwich enzyme-linked immunosorbent assay (ELISA) using the solid phase and alkaline phosphatase-linked monoclonal antibody $(\mathrm{mAb}) \mathrm{G11}$. mAb Gl1 was also used to determine the localization of the Em2 antigen by immunofluorescence and post-embedding immunogold electron microscopy.

6. Finally, the infectiosness of in vitro-cultivated vesicles was proven in vivo by experimental infection of recipient host mice.

\section{Parasite}

A cloned isolate of $E$. multilocularis, clone KF5 (Gottstein et al. 1992), was maintained in $\mathrm{C} 57 \mathrm{BL} / 10$ or $\mathrm{C} 57 \mathrm{BL} / 6$ mice by peritoneal injection of $50 \mu \mathrm{l}$ of a vesicle suspension. Mice were euthanized with $\mathrm{CO}_{2}$ and metacestode material was recovered under aseptic conditions from the peritoneal cavity. Vesicle suspensions were prepared by pressing parasite material through a sterile metal sieve (0.5-mm diameter mesh size). Having passed the sieve, the small parasite vesicles were washed twice with Hanks' balanced salt solution (HBSS) and were resuspended in RPMI 1640. They were used for subsequent intraperitoneal infections employing a 1-ml syringe with an 18G11/2-needle. The same procedure was applied to inoculate in vitro-grown metacestode vesicles of less than $0.5 \mathrm{~mm}$ diameter for proof of infectiosness.

\section{Sera}

Anti- $E$. multilocularis immune serum was obtained from a Swiss man with histologically proven $\mathrm{AE}$ of the liver; blood was drawn prior to treatment. Anti-E. granulosus immune serum was obtained from an Italian women with a histologically proven hepatic hydatid cyst; blood was drawn prior to treatment. Negative control serum was arbitrarily selected from a serum bank of healthy Swiss blood donors.
$\mathrm{CACO} 2$ cells

Human cancer colon cells (CACO2) were maintained in $25-\mathrm{mm}^{2}$ tissue-culture flasks in RPMI 1640 medium containing $12 \mathrm{mM}$ HEPES and supplemented with $10 \%$ fetal calf serum (FCS), $2 \mathrm{mM}$ glutamine, $100 \mathrm{U}$ penicillin $/ \mathrm{ml}$, and $100 \mu \mathrm{g}$ streptomycin $/ \mathrm{ml}$ at $37^{\circ} \mathrm{C}$ in an atmosphere containg $5 \% \mathrm{CO}_{2}$.

\section{In vitro cultivation of $E$. multilocularis metacestodes}

Freshly recovered metacestode tissue was cut into small tissue blocks $\left(0.5 \mathrm{~cm}^{3}\right)$ and washed twice in HBSS, after which two pieces of each tissue were placed in $40 \mathrm{ml}$ of RPMI 1640 medium containing $12 \mathrm{~m} M$ HEPES, $10 \% \mathrm{FCS}, 2 \mathrm{~m} M$ glutamine, $200 \mathrm{U}$ of penicillin/ml, $200 \mu \mathrm{g}$ streptomycin $/ \mathrm{ml}, 0.50 \mu \mathrm{g}$ fungizone $/ \mathrm{ml}$ and $50 \mu M \beta$-mercaptoethanol. Alternatively, vesicle suspensions were prepared and placed in $40 \mathrm{ml}$ of culture medium as described above. Incubations were performed in tightly closed tissue-culture flasks $\left(25 \mathrm{~mm}^{2}\right)$ placed in the upright position in an incubator containing $5 \% \mathrm{CO}_{2}$ at $37^{\circ} \mathrm{C}$ for a period of 100 days, with medium changes occurring every 3-4 days. Before each change of medium, the number of vesicles, the vesicle diameters, and the number of vesicles harboring protoscolices were determined. We studied the vesicle growth and proliferation of three cultures initiated from tissue blocks and of three initiated from vesicle suspensions, all originating from the same $\mathrm{C} 57 \mathrm{BL} / 10$ mouse. The results obtained from two cultures of each are presented.

For some experiments, metacestodes were cultured in serumfree medium. Vesicles (3- to 5- $\mathrm{mm}$ diameter) were carefully removed from their original culture flask. They were washed eight to ten times in sterile phosphate-buffered saline (PBS) to remove the FCS. Washed vesicles were transferred to a tissue-culture flask containing serum-free medium and were kept at $37^{\circ} \mathrm{C}$ in an atmosphere containing $5 \% \mathrm{CO}_{2}$ for $7-10$ days.

E. multilocularis cultures containing $\mathrm{CACO} 2$ feeder cells were initiated by first growing $\mathrm{CACO} 2$ cells to confluency. The flask was then brought to the upright position, $40 \mathrm{ml}$ of metacestode medium was added, and a defined number of in vitro-generated metacestode vesicles (3- to 5-mm diameter) were carefully transferred into the flask. As a control, an identical number of vesicles of similar sizes were transferred to a tissue flask devoid of feeder cells. Culturing and counting of these feeder-cell cultures was performed as described above. Care had to be taken not to destroy the feeder-cell layer physically during medium changes. Every 3-4 weeks, the feeder-cell culture-derived vesicles were tranferred into a flask with a fresh $\mathrm{CACO} 2$ cell layer. We studied the vesicle growth and proliferation of three feeder-cell cultures of tissueblock- and vesicle-suspension-derived metacestodes, all providing the same result.

\section{Isolation of parasite fractions}

Crude E. multilocularis metacestode extracts (clones F5 and FAub) were prepared according to Gottstein et al. (1983). In vitrogenerated metacestodes cultured in either serum-free medium, medium+FCS, or medium+FCS+CACO2 feeder cells were transferred into $15-\mathrm{ml}$ centrifuge tubes. The medium supernatants of each culture were collected and frozen at $-80^{\circ} \mathrm{C}$ until further used. The vesicles were carefully washed eight to ten times with PBS. As vesicles were floating freely in the culture medium, they could be rapidly sedimented at $1 \mathrm{~g}$ by adding an equal volume of sterile $\mathrm{H}_{2} \mathrm{O}$ to the medium. Alternatively, they were purified efficiently by flotation at $1 \mathrm{~g}$ by mixing $20 \%(\mathrm{v} / \mathrm{v})$ of Ficoll Hypaque (Pharmacia) to the culture medium. Metacestode vesicles were then broken up by gentle disruption using a glass pipette, and the metacestode tissue (composed of the laminated layer, tegument, and germinal layer) was separated from the vesicle fluid by centrifugation at $10,000 \mathrm{~g}$ for $20 \mathrm{~min}$ at $4^{\circ} \mathrm{C}$. The supernatants (i.e., vesicle fluids) were carefully removed by aspiration and concentrated in a Centricon 10 microconcentrator (Amicon) according to 
the instructions provided by the manufacturers. Protein concentrations were determined with the BioRad protein assay. Vesicle fluids were stored at $-80^{\circ} \mathrm{C}$. The pellets containing the vesicle walls were resuspended in PBS and sonicated, and samples were taken for measurement of the protein concentrations. These extracts were also stored at $-80^{\circ} \mathrm{C}$ prior to their use.

\section{Immunoblotting and Em2 ELISA}

Crude extracts of metacestode clone F5 and in vitro-generated vesicle walls $(1 \mu \mathrm{g} / \mathrm{ml}$ each) as well as vesicle fluids $(0.5 \mu \mathrm{g} / \mathrm{ml})$ were solubilized in sodium dodecyl sulfate (SDS) sample buffer and processed for SDS-polyacrylamide gel electrophoresis (SDSPAGE) and immunoblotting as previously described (Gottstein et al. 1986). Serum dilutions and conjugates were as previously described (Gottstein et al. 1994). Visualization of immunoreactive bands was performed according to Dao (1985). The double sandwich ELISA for the detection of Em2 antigen in vesicle fluids and walls and in medium supernatants corresponded exactly to the one described by Deplazes and Gottstein (1991). As a control, identical experiments were performed with the cestode-irrelevant $\mathrm{mAb}$ G10/4 of the same isotype (Aggarwal et al. 1989).

\section{Lymphocyte proliferation assay}

Peripheral blood monocytes (PBMC) were obtained from heparinized whole blood of an $\mathrm{AE}$ patient and a healthy control individual using lymphocyte separation medium (Ficoll-Paque, Pharmacia). Lymphocytes were washed twice with HBSS and the numbers of viable cells were determined by trypan blue exclusion. Isolated cells were resuspended at a concentration of $0.5 \times 10^{6} / \mathrm{mI}$ in RPMI containing $12 \mathrm{~m} M$ HEPES and supplemented with $2 \mathrm{~m} M$ glutamine, $100 \mathrm{U}$ penicillin $/ \mathrm{ml}$, and $100 \mu \mathrm{g}$ streptomycin $/ \mathrm{ml}$. Cells were plated out into 96 -well plates $(200 \mu \mathrm{l} /$ well $)$. Antigen stimulation was performed with $10 \mu \mathrm{g}$ of each extract per well. As an internal stimulation control, $0.25 \mu \mathrm{g}$ phytohemagglutinin (PHA)/well was used in triplicate determinations. On the 3rd day of stimulation, cells were pulsed with $1 \mu \mathrm{Ci} /$ well and subsequently harvested after $16-18 \mathrm{~h}$.

\section{Morphology and ultrastructure}

\section{Scanning electron microscopy}

In vitro-generated secondary vesicles were gently broken up to release the vesicle fluid. They were fixed in $2.5 \%$ glutaraldehyde diluted in $100 \mathrm{mM}$ sodium phosphate buffer ( $\mathrm{pH} \mathrm{7.2)} \mathrm{for} 4 \mathrm{~h}$ at $4^{\circ} \mathrm{C}$. Parasite material was then washed three times in phosphate buffer. Postfixation took place in $2 \%$ osmium tetroxide for $2 \mathrm{~h}$ at $4^{\circ} \mathrm{C}$. After three additional washes in distilled water, metacestodes were dehydrated through a graded series of acetone. They were then dried with Peldri II, a fluorocarbon compound that was used as an alternative to critical-point drying (Kennedy et al. 1989). Specimens were then sputter-coated with gold, and inspection took place on a Jeol 840 scanning electron microscope (SEM) operating at $25 \mathrm{kV}$.

\section{Transmission electron microscopy}

Vesicles were fixed for $4 \mathrm{~h}$ at $4^{\circ} \mathrm{C}$ in $2.5 \%$ glutaraldehyde diluted in $100 \mathrm{~m} M$ phosphate buffer ( $\mathrm{pH} 7.2$ ) supplemented with $0.25 \%$ tannic acid (Mallinkrodt, USA). After several washes in $100 \mathrm{mM}$ phosphate buffer, they were postfixed in $2 \%$ osmium tetroxide for $2 \mathrm{~h}$ and rinsed several times in distilled water. Subsequent incubation in $1 \%$ uranyl acetate in water was carried out for $1 \mathrm{~h}$ at $4^{\circ} \mathrm{C}$. Metacestodes were then dehydrated through a graded series of ethanol and embedded in Epon 812 resin (Fluka). Polymerization took place at $65^{\circ} \mathrm{C}$ for $24-36 \mathrm{~h}$. Ultrathin sections were cut on a
Reichert and Jung ultramicrotome. Sections were picked up on 200-mesh copper or nickel grids. Staining with uranyl acetate and lead citrate was performed as described elesewhere (Smith and Croft 1991). Specimens were viewed and photographed on a Phillips 600 transmission electron microscope (TEM) operating at $60 \mathrm{kV}$.

Immunocytochemistry

\section{Direct immunofluorescence}

Specific identification of the Em2-positive laminated layer was carried out using the direct immunofluorescence technique with fluorescein isothiocyanate (FITC)-labeled mAb G11 as described in Deplazes and Gottstein (1991).

\section{Fixation and embedding for postembedding immunocytochemistry}

For immunocytochemical localization of the Em2 antigen on sections of in vitro-generated metacestodes, vesicles were embedded in LR-White (Sigma). Fixation took place in $100 \mathrm{~m} M$ phosphate buffer containing $3 \%$ paraformaldehyde $/ 0.1 \%$ glutaraldehyde for $40 \mathrm{~min}$ at $4^{\circ} \mathrm{C}$. Metacestode tissue was then rinsed twice in PBS, and free aldehyde groups were saturated with $50 \mathrm{mM}$ glycine in PBS for $30 \mathrm{~min}$ at room temperature. After three washes in PBS, specimens were dehydrated through $50 \%, 70 \%$, and $90 \%$ ethanol for $10 \mathrm{~min}$ each and underwent three washes in $100 \%$ ethanol for 15 min each. All dehydration steps were carried out at $-20^{\circ} \mathrm{C}$. Metacestodes were infiltrated with four changes of LR-White during a $48-\mathrm{h}$ period at $-20^{\circ} \mathrm{C}$. The resin was then polymerized at $60^{\circ} \mathrm{C}$ for $8-12 \mathrm{~h}$.

\section{Indirect immunofluorescence}

Sections of $1 \mu \mathrm{m}$ for $\mathrm{mAbG11}$ staining were loaded onto poly-Llysine-coated coverslips and were air dried. Sections were incubated overnight in PBS supplemented with $3 \%$ bovine serum albumin (BSA), $100 \mathrm{mM}$ glycine, and $0.05 \%$ Tween- 20 at $4^{\circ} \mathrm{C}$. They were then rinsed in PBS and incubated for $1 \mathrm{~h}$ with $\mathrm{mAb}$ G11 diluted $1: 100$ in $\mathrm{PBS} / 0.5 \% \mathrm{BSA} / 50 \mathrm{~m} M$ glycine/ $0.05 \%$ Tween- 20 (antibody buffer) at room temperature. As a control, an irrevelant $\mathrm{mAb}$ (G10/4) of the same isotype (IgG1) was used. Coverslips were then washed four times for $5 \mathrm{~min}$ in PBS, and FITC-conjugated goat anti-mouse (Cappel) was applied at a dilution of 1:100 in antibody buffer for $1 \mathrm{~h}$. Subsequently, the coverslips were washed six times for $5 \mathrm{~min}$ in PBS before being embedded in a mixture of Gelvatol/glycerol containing 1,4-Diazobicyclo (2.2.2.) octan (Merck AG, Zürich) to prevent fading of the FITC (Hemphill et al. 1992). They were viewed on a Leitz Laborlux $S$ fluorescence microscope.

\section{Immunogold labeling}

Ultrathin sections were loaded onto carbon/Formvar-coated nickel grids (Agar Scientific Ltd, UK), air-dried, and further processed within 2-3 $\mathrm{h}$. Blocking of unspecific binding sites and exposure to $\mathrm{mAb}$ G11 was performed as described above. Incubation with a goat anti-mouse Ig antibody conjugated to $10-\mathrm{nm}$ gold particles (Amersham Rahn, Zürich) was carried out at a dilution of $1: 10$ in PBS/0.1\%BSA for $1 \mathrm{~h}$ at room temperature. After six washes in PBS ( 5 min each), grids were rinsed three times in distilled water and were subsequently air-dried. They were finally stained with uranyl acetate and lead citrate as described above. 


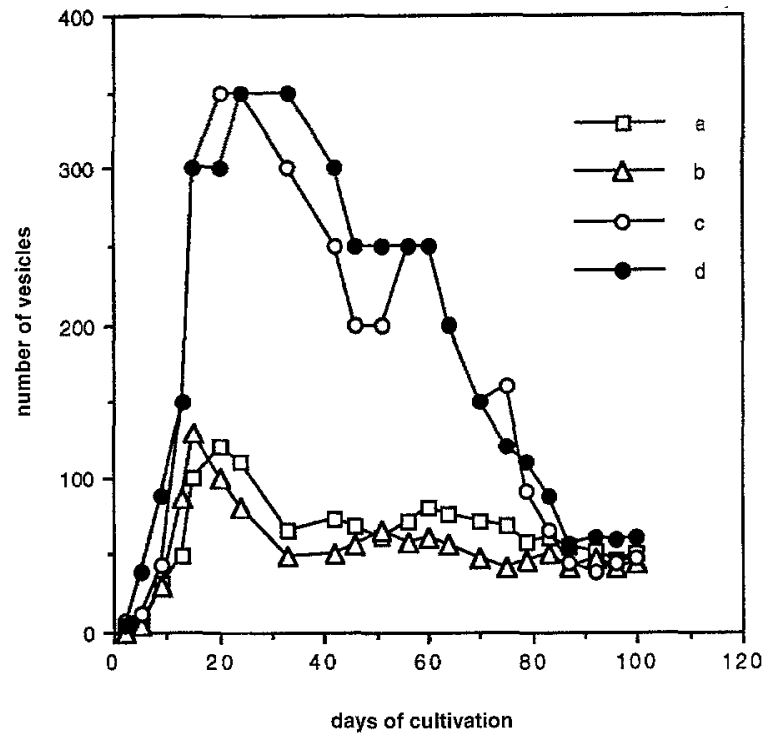

Fig. 1 In vitro cultivation of Echinococcus multilocularis metacestodes during a time span of 100 days, respective to the number of vesicles synthesised ( $a, b$ Cultures originating from tissueblocks, $c, d$ cultures originating from vesicle suspensions)

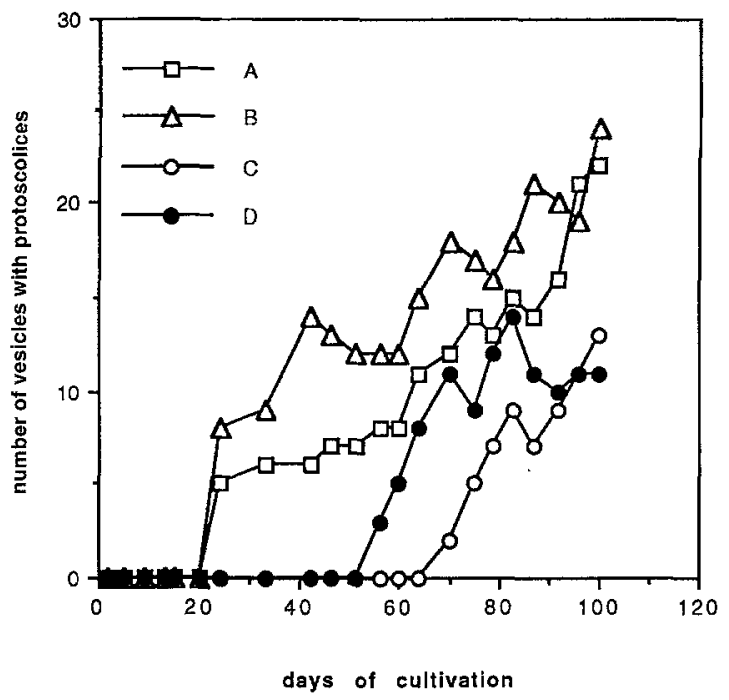

Fig. 2 Occurrence and number of vesicles developing protoscolices during in vitro cultivation of $E$. multilocularis metacestodes ( $A, B$ Vesicles originating from vesicle suspensions, $C, D$ vesicles from tissue-block-derived cultures)

\section{Results}

In vitro cultivation of Echinococcus multilocularis metacestodes

E. multilocularis metacestodes recovered from experimentally infected mice were used to establish two types of cultures, namely, (1) tissue-block-derived cultures (Fig. 1, graphs a, b) and (2) vesicle-suspension-derived cultures (Fig. 1, graphs c, d). Metacestode cultivation over a period of 100 days in both types of culture was

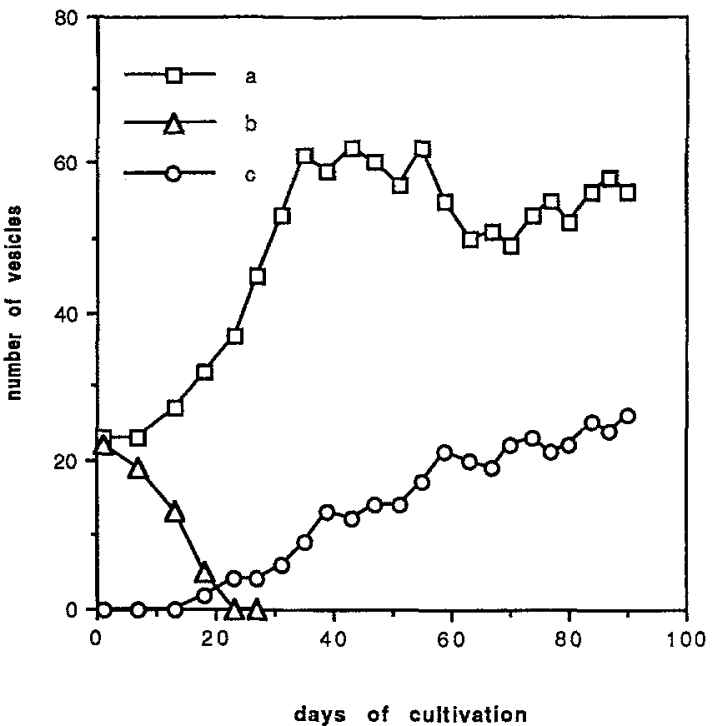

Fig. 3 In vitro cultivation of E. multilocularis metacestodes (respective to the number of vesicles synthesised) in the presence $(a)$ or absence $(b)$ of CACO2 feeder-cells $(c)$ Number of metacestodes exhibiting protoscolex formation in the culture relative to $(a)$

characterized by three distinct phases: (1) an initially accelerated progression of vesicle growth, (2) a subsequent regression in vesicle numbers, and (3) a final phase of stable vesicle production.

Proliferation of metacestodes took place by budding of small $(<0.5-\mathrm{mm}$ diameter) daughter vesicles either from larger parent vesicles or directly from the tissue block (data not shown). During the first 20 days of cultivation, metacestode production in vesicle-suspension-derived cultures was about 3-fold that in tissue-block-derived cultures (Fig. 1); however, their vesicle numbers diminished dramatically during the course of cultivation (from day 20 to day 80 ), and after 90 days they reached levels similar to those observed in tissue-block-derived cultures. In contrast, cultures initiated from small blocks of parasite tissue exhibited a less dramatic progression and subsequent regression in terms of vesicle numbers, and after about 35 days of in vitro cultivation the number of metacestodes in these tissue-block-derived culture types remained stable over a period of 65 days (Fig. 1).

Growth of individual vesicles was also recorded (data not shown). In general, tissue-block-derived cultures produced larger vesicles than did suspension-derived cultures. At the end of the cultivation period of 100 days, vesicle diameters had reached $16 \mathrm{~mm}$ in tissue-block-derived cultures and $12 \mathrm{~mm}$ in suspension-derived cultures.

In addition, we observed significant differences with respect to protoscolex development (Fig. 2). Suspensionderived metacestodes started to develop protoscolices after approx. 24 days of in vitro cultivation, whereas tissue-block-derived parasites were not fertile until days 49-70. There was no obvious correlation between the development of protoscolices and the size of the metacestodes. Although larger vesicles $(>10 \mathrm{~mm})$ produced a higher number of developing protoscolices, we found 
Fig. 4-6 Light and electron microscopy of in vitro-cultivated metacestodes. Fig. 4a Light microscopical view of a small (<3 mm) vesicle harboring developing protoscolices. Fig. $4 \mathrm{~b}$ Corresponding direct immunofluorescence surface labeling of FITC-labeled $\mathrm{mAb}$ G11, which reacts specifically with the Em2 antigen. Fig. 5 SEM of a fertile metacestode, demonstrating the smooth outer surface comprising the laminated layer $(L L)$ and the inner, germinal layer $(G)$ with germinating protoscolices $(B)$.

Bar $=380 \mu \mathrm{m}$. Fig. 6 TEM of the metacestode wall, demonstrating the acellular laminated layer $(L L)$ and the cellular germinal (G) layer (te Tegument, $m t$ microtriches, uc undifferentiated cell, $g c$ glycogen storage cell, $m c$ muscle cell).

$\mathrm{Bar}=2.6 \mu \mathrm{m}$
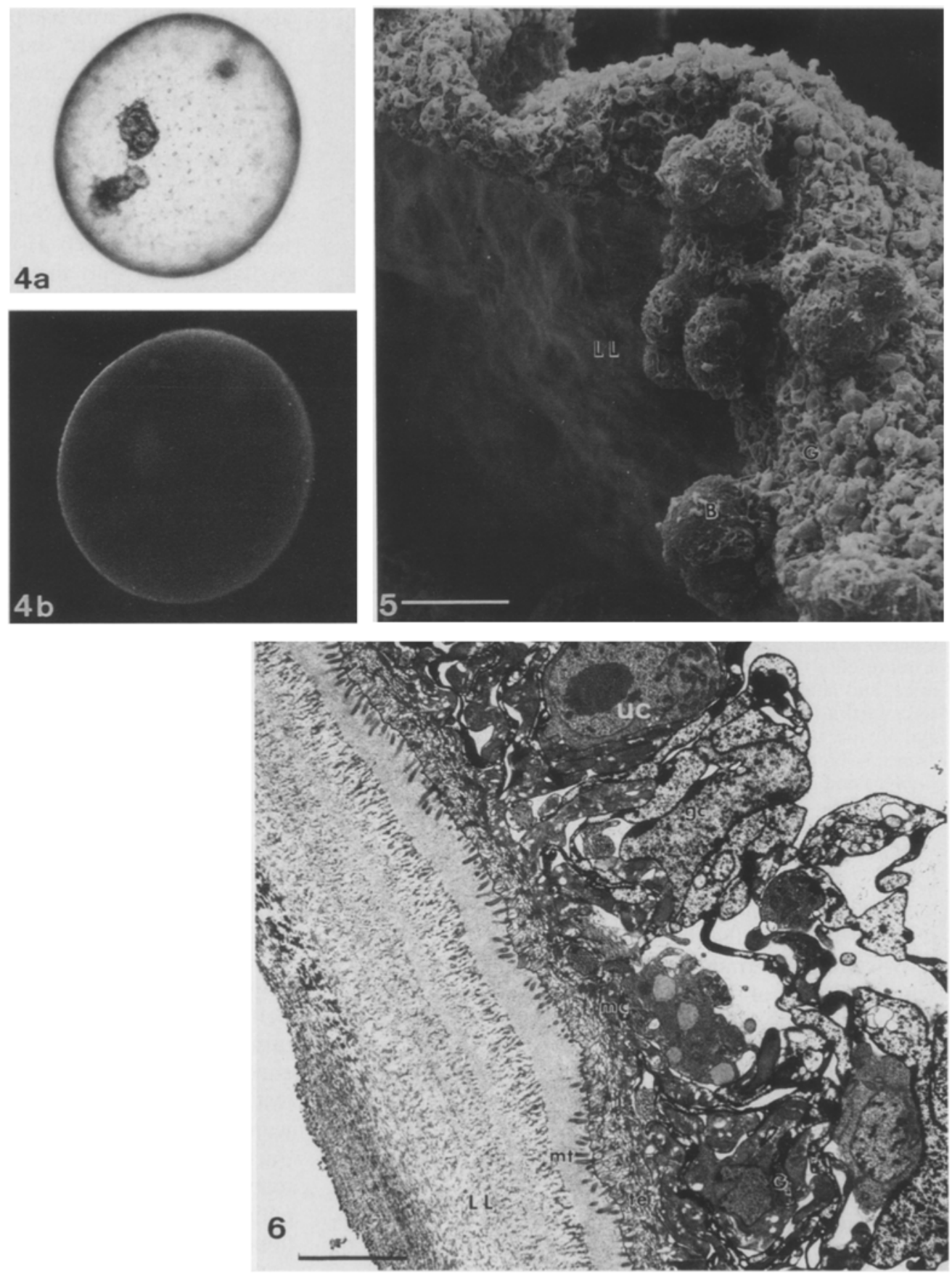

vesicles with diameters of below $3 \mathrm{~mm}$ that were also producing protoscolices, albeit in lower numbers (see Fig. 4a).

After 35 days of in vitro cultivation, small vesicles $(<0.5 \mathrm{~mm})$ were collected and used for intraperitoneal infection of $\mathrm{C} 57 \mathrm{BL} / 10$ and $\mathrm{C} 57 \mathrm{BL} / 6$ mice. At 3 months after infection it was found that these in vitro-generated metacestodes were capable of inducing secondary murine AE. Whereas in necropsied "resistant" C57BL/10 mice, no protoscolex was found, the parasite was producing large amounts of protoscolices in susceptible C57BL/6 mice (data not shown). A comparison of the morphology of in vitro-generated protoscolices with that of protoscolices isolated from an infected C57BL/6 mouse by both light and scanning electron microscopy revealed that there was no morphological difference between the two (data not shown).

\section{CACO2 feeder-cell cultures}

For assessment of the influence of heterologous host cells on parasite growth and development of protoscolices, 22 vesicles ( 3 to 5 -mm diameter) were collected from an original tissue-block- or vesicle-suspension-derived culture and were incubated in the presence or absence of 


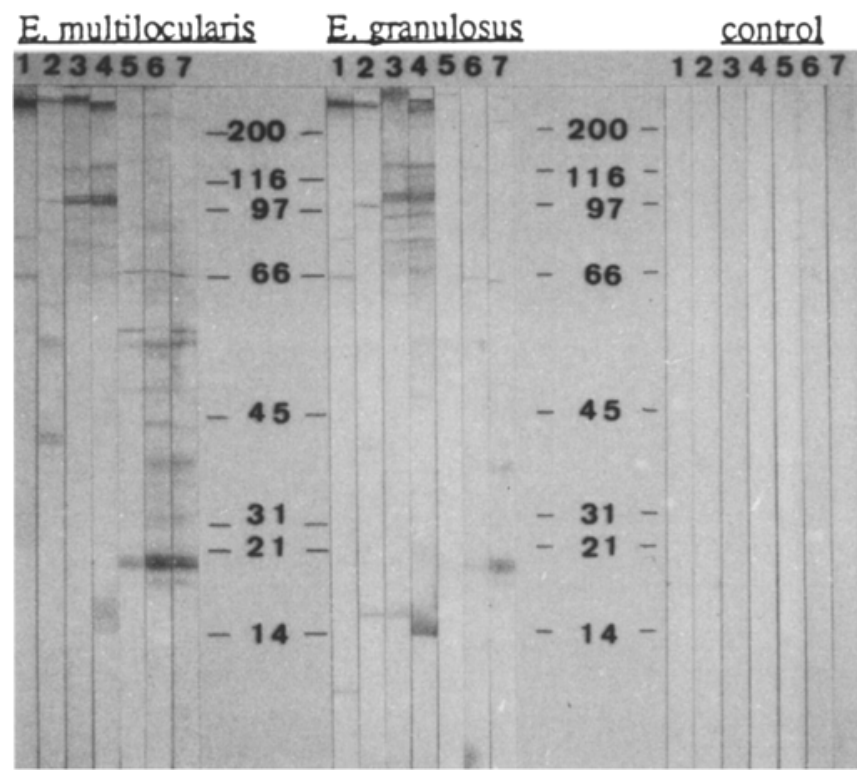

Fig. 7 Immunoblots of metacestode fractions reacting with sera from an $E$. multilocularis- and an $E$. granulosus-infected patient. Control strips are labeled with serum from a healthy person. Lane 1 represents the metacestode extract of clone F5 generated in mice, and lanes 2-4 show in vitro-generated vesicle fluids of cultures grown in the absence (lane 2) and presence (lane 3 ) of FCS and in the presence of FCS and CACO2 feeder-cells (lane 4). Lanes 5-7 represent metacestode extracts generated in the absence (lane 5) and presence (lane 6) of FCS and in the presence of feeder-cells (lane 7)

$\mathrm{CACO} 2$ feeder cells. The results were identical for metacestodes from both culture types (Fig. 3). In the absence of feeder cells, the metacestodes diminished rapidly in size, proliferation ceased, and parasites were dead within 25 days of cultivation. In contrast, feeder-cell cultures remained viable for several months as suggested by the proliferation and growth of vesicles as well as the development of protoscolices after 12 days of cultivation in the presence of feeder cells. Due to the CACO2 feedercell layer, metacestode cultures remained viable for at least 90 days postinoculation (Fig. 3). These results suggest that the heterologous $\mathrm{CACO} 2$ cell line produces growth factors and/or other molecules that promote parasite viability, growth, and proliferation as well as the production of protoscolices in vitro. Cytotoxic effects of $E$. multilocularis metacestodes on their $\mathrm{CACO} 2$ feedercells were not observed (data not shown).

\section{Morphology of in vitro-cultivated $E$. multilocularis metacestodes}

The morphology of in vitro-generated metacestodes was investigated light microscopically (Fig. 4a) and by scanning (SEM, Fig. 5) and transmission (TEM, Fig. 6) electron microscopy. Identical results were obtained from tissue-block-derived, vesicle-suspension-derived, and $\mathrm{CACO} 2$ feeder-cell cultures. The surface of the metacestodes accessible from the outside had a smooth appear- ance (Fig. 5). It was composed of the acellular laminated layer, which covered the entire larva. The tissue inside contained the multicellular germinal layer and, in fertile metacestodes, immature structures harboring the developing protoscolices. The latter were in several cases partially evaginated upon maturation. TEM of metacestodes revealed that the general features of these in vitro-generated parasites were identical to those of metacestodes found in vivo (Fig. 6). However, due to the incorporation of tannic acid into the fixation protocol, the acellular, carbohydrate-rich laminated layer remained well preserved and showed a unique structural organization. It appeared to consist of several layers with different architectural features. The most outer cellular layer of the metacestode was the syncytial tegument, with its microtriches protruding well into the laminated layer. The germinal layer consisted of connective tissue, muscle cells, glycogen-containing storage cells, and undifferentiated cells.

Antigenic profiles of in vitro-generated metacestode fractions

To obtain information on the expression of B-cell epitopes by in vitro-cultivated $E$. multilocularis metacestodes, we performed immunoblotting using E. multilocularis- and E. granulosus-infected patients' sera. Figure 7 shows immunoblots of crude in vivo-generated $E$. multilocularis extract (clone F5), of vesicle fluids, and of cellular metacestode extracts generated from in vitro cultures grown in the presence or absence of FCS or in the presence or absence of $\mathrm{CACO} 2$ feeder-cells. Both $E$. multilocularis- and E. granulosus-infected patients' sera contained antibodies against antigens present in all fractions; no immunoreactivity was found in the control serum from a healthy individual. However, distinct differences in banding patterns were observed in all extracts tested. The differences between the two patients' sera were most pronounced in the cellular metacestode extracts (Fig. 7, lanes 5-7), with quantitatively more bands reacting with the serum of the $E$. multilocularis-infected patient. Furthermore, the E. granulosus-infected patients' serum recognized a distinct additional band of low molecular weight in in vitro-generated vesicle fluids (lanes 2-4). We concluded from these immunoblots that in vitro-cultivated metacestodes synthesized molecules that represented B-cell epitopes in infected patients suffering from both $\mathrm{AE}$ and hydatid disease.

Assessment of T-cell epitope expression on in vitrogenerated metacestodes was performed by testing equal amounts of various antigens for their potential to induce the proliferation of lymphocytes from a patient suffering from $\mathrm{AE}$. Besides performing this assay with crude metacestode extracts (obtained with clone F5 and isolate FAub grown in mice) as a positive reference control (Gottstein et al. 1994), we comparatively analyzed in vitro-generated vesicle fluids and cellular parasite extracts as well as the corresponding medium supernatants. As 
Fig. 8 Lymphocyte proliferation assay using human PBMC from a patient with alveolar echinococcosis. PBMC were stimulated in vivo with metacestode extracts (clones F5 and isolate FAub2) and vesicle fluids, vesicle walls, and medium supernatants from parasites cultivated in vitro under different conditions

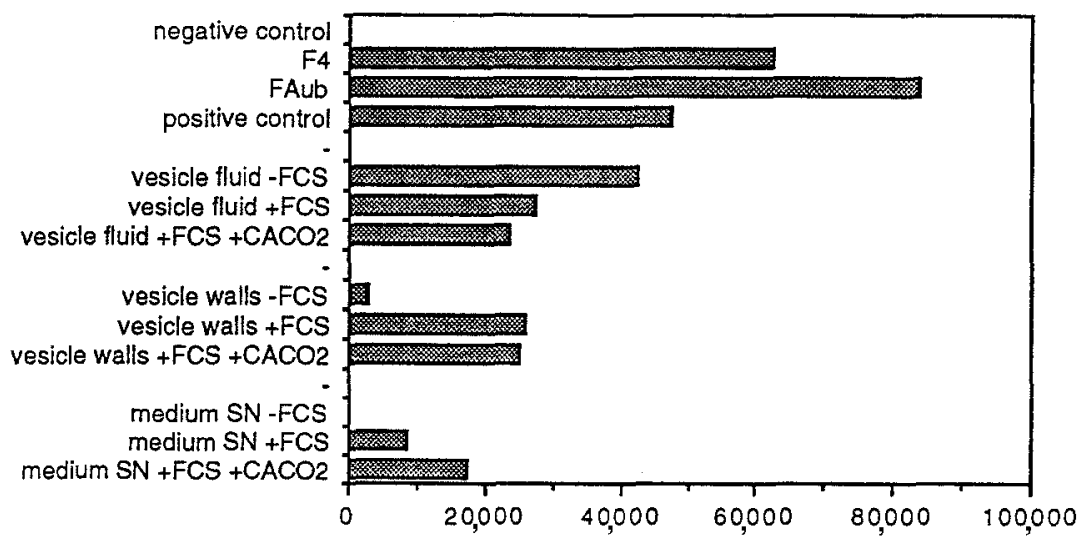

[ ${ }_{3} \mathrm{H}$ ]-thymidine incorporation in cpm

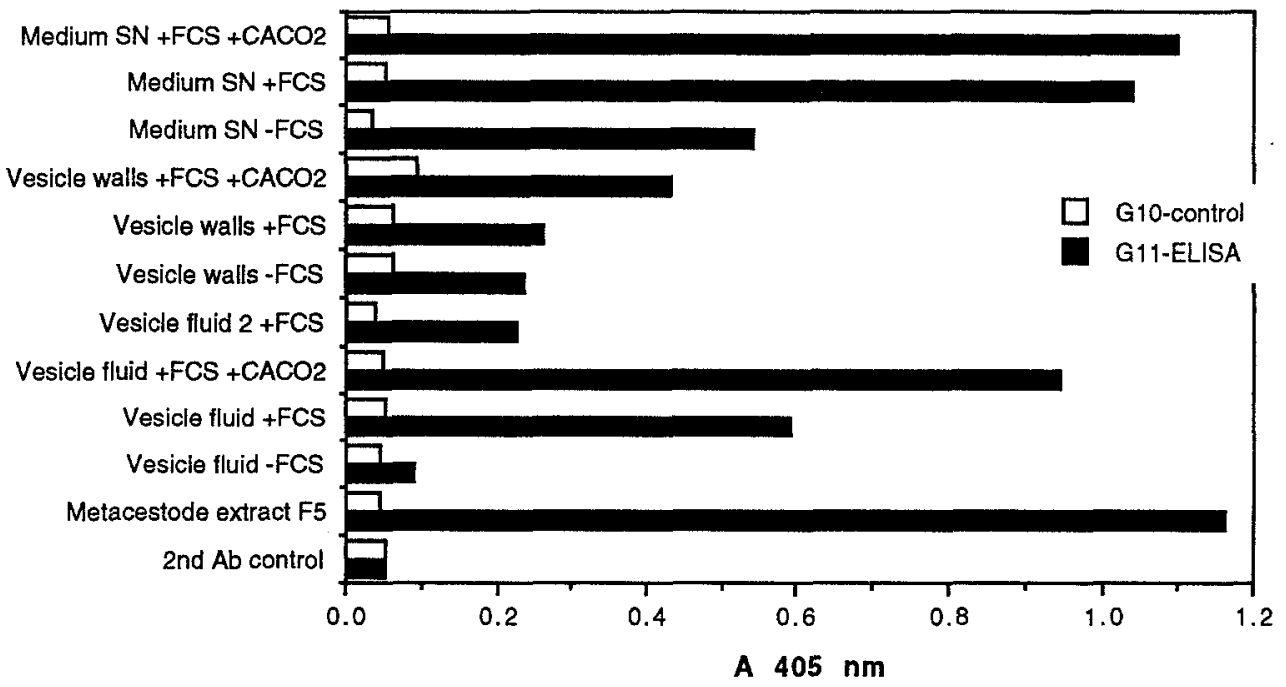

Fig. 9 Double-antibody sandwich ELISA demonstrating the presence of Em2 antigen in vivo (metacestode extract F5) and in in vitro-grown vesicle fluids, vesicle walls, and medium supernatants. As a control, incubations were also performed with an irrelevant control $\mathrm{mAb}(\mathrm{G} 10)$ of the same isotype at the same concentration
FITC so as to test the surface accessibility of the Em2 antigen on in vitro-generated metacestodes. Figure $4 b$ demonstrates the presence of this antigen on the surface of the parasite larvae.

For acquisition of additional information on the localization of the Em2 antigen in in vitro-cultured $E$. multilocularis metacestodes, parasites were embedded in LRWhite and $1-\mu \mathrm{m}$ sections were stained with mAb G11 and an appropriate FITC-conjugated second antibody (Fig. 10). Inspection of these stained sections revealed that the localization of the Em2 antigen was restricted to the area of the vesicle laminated layer. Neither the germinal layer nor the immature or mature protoscolices were labeled with $\mathrm{mAb}$ G11.

To localize the Em2 antigen more precisely, we carried out electron microscope investigations using LRWhite-embedded metacestodes, mAb G11, and a second antibody conjugated to $10-\mathrm{nm}$ gold particles as an electron-dense marker (Fig. 11). The presence of the Em2 antigen was almost entirely restricted to the laminated layer. Very few gold particles were found on the tegument, and labeling was entirely absent on all other parts of the parasite. Em2 was also associated with the tegumental microtriches, which extended considerably into 
Fig. 10,11 Localization of the Em2 antigen using $\mathrm{mAb}$ G11.

Fig. 10 a Light micrograph and b corresponding indirect immunofluorescence obtained with ed second antibody on LRWhite sections. Staining is present on the periphery of the metacestode $(P)$ but is not visible on proliferating protoscolices $(B)$ or on the germinal layer $(G L)$. Fig. 11 mAb G11 immunogold labeling on a section of the same block shown in

Fig. 10. Gold particles are ing is observed in the region surrounding the microtriches $(M)$, and gold particles are also found on the outer surface of the laminated layer $(O S)$. $\mathrm{Bar}=1.5 \mu \mathrm{m}$ mAb G11 and FITC-conjugatfound exclusively on the laminated layer $(L L)$. Dense label-
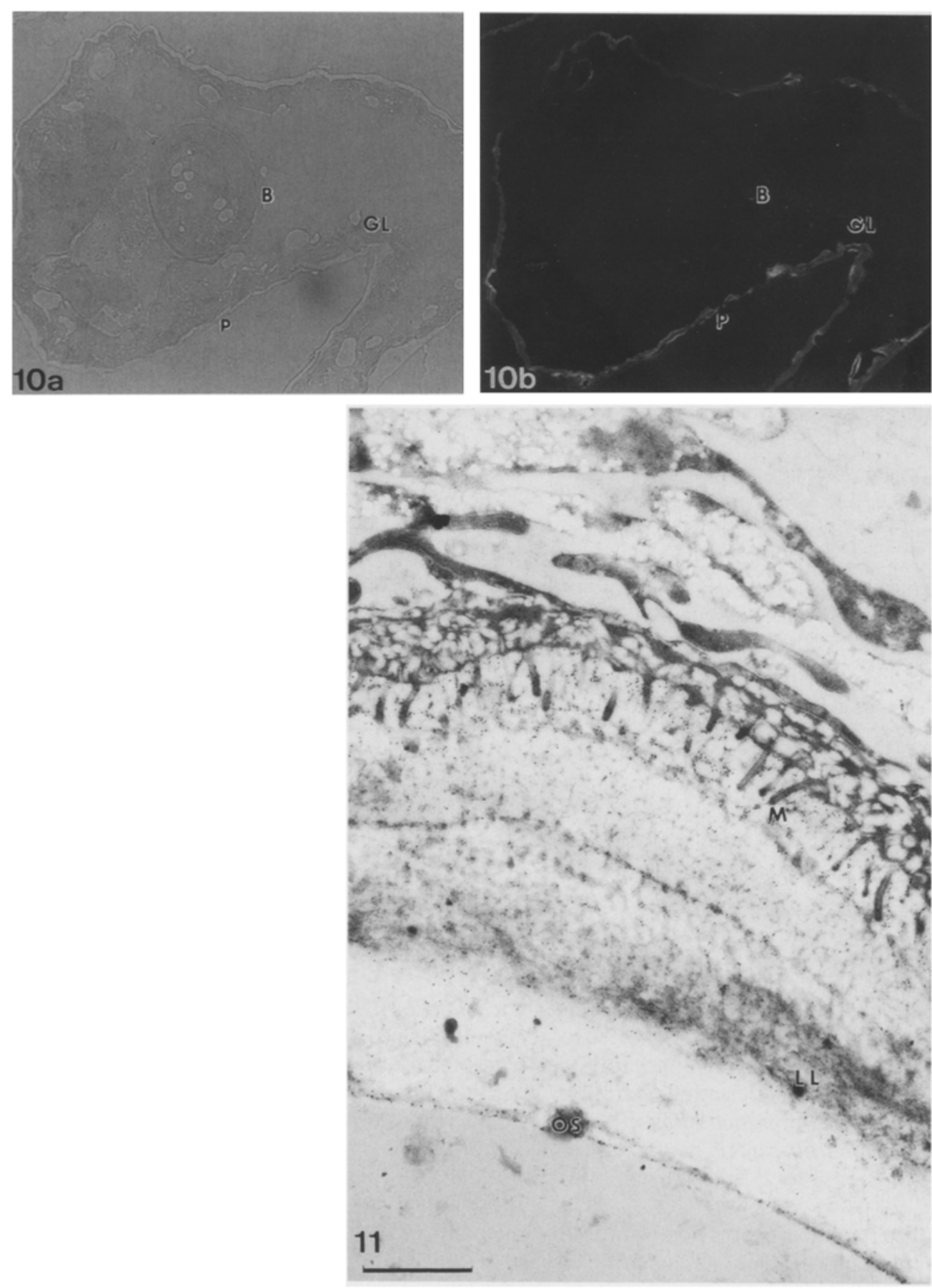

the laminated layer. In addition, gold particles were found along the outer surface of the laminated layer.

\section{Discussion}

The present study presents a newly established cultivation method for maintenance and proliferation of the larval stage of Echinococcus multilocularis in vitro. Cultures were initiated either from small blocks of infected tissue or from vesicle suspensions. As determined using defined culture conditions, metacestodes proliferated, in- creased in size, and exhibited fertility with respect to protoscolex development.

Proliferation of metacestodes under the culture conditions used for this study was characteristically divided into three phases, namely, rapid proliferation within the first 20-25 days of cultivation, subsequent regression, and, finally, stabilization of metacestode numbers. Proliferation took place by budding of small daughter vesicles from larger parent vesicles. A similar mechanism, namely, continous exogenous budding with progressive invasion of the surrounding tissue, has been suggested to be responsible for metastasis formation in rodents and 
gerbils (Eckert et al. 1983; Melhorn et al. 1983). We also found that during the initial phase of in vitro cultivation, cultures originating from vesicle suspensions were metabolically much more active in terms of vesicle production than were tissue-block-derived cultures. It is likely that this resulted from the higher number of small vesicles actually liberated during preparation of the suspension. These liberated vesicles were not physically encapsulated by growth-inhibiting connective tissue; thus, they were capable of proliferating at a higher rate. Interestingly, after 80-90 days of cultivation the number of metacestodes in both culture types stabilized at around 50-60 per flask.

However, incubation of considerably lower numbers of metacestodes under similar conditions resulted in the death of these parasites within 25 days. Thus, a minimal concentration of vesicles was necessary to sustain survival in vitro, probably due to a critical concentration of metabolic products and/or growth factors synthesized by the parasites themselves. We found that factors necessary for growth, proliferation, and protoscolex formation could also be provided by heterologous $\mathrm{CACO} 2$ feedercells. Although the increase in vesicle numbers took place rather gradually and not at all as dramatically as in the original cultures, the number of vesicles observed after a prolonged cultivation period (90 days) was similar to the number of metacestodes seen in original cultures after 100 days in vitro.

The pathogenicity of in vitro-generated metacestodes from both culture types was confirmed by infection and induction of murine $\mathrm{AE}$ in both susceptible $\mathrm{C} 57 \mathrm{BL} / 6$ and relatively "resistant" C57BL/10 mice (Gottstein et al. 1994). As expected, protoscolex formation was induced in the susceptible mouse strain but did not occur in the "resistant" one. Wheras protoscolex formation has only rarely been observed in human $\mathrm{AE}$ (Gottstein et al. 1992), the development of protoscolices in rodents usually depends strongly on intermediate host species and strains (Thompson and Lymbery 1988) and usually takes place within 2-4 months of infection. We have also monitored protoscolex development during in vitro cultivation. Probably due to their increased metabolic activity, the development of protoscolices started much earlier in vesicle-suspension-derived cultures (around day 20) than in tissue-block-derived cultures (days 60-70). However, at the end of the cultivation period of 100 days, these protoscolices were morphologically identical to in vivogenerated parasites isolated from an infected C57BL/6 mouse.

The morphology of metacetodes generated in vitro as well as in vivo (separated from the host in a micropore chamber; Nahhas et al. 1991) was very similar to that of metacestodes isolated from the peritoneum of experimentally infected mice and gerbils (Delabre et al. 1987; Mehlhorn et al. 1983). The parasite tissue was surrounded by the acellular laminated layer, a structure that is known to play a decisive role in protecting the parasite from its host's immune reaction (Alkarmi et al. 1988; Gottstein et al. 1992, 1994). That this laminated layer was always present during cultivation in vitro clearly indicated that this structure was synthesized by the parasite itself. However, the possibility has to be considered that in vivo, host material may contribute to the formation of the laminated layer of E. multilocularis (Mehlhorn et al. 1983). The most outer cellular layer is composed of the syncytial tegument followed by connective and muscle tissue, cells containing glycogen, and undifferentiated cells, in a way very similar to that previously observed in tissue infected by E. multilocularis (Delabre et al. 1987; Mehlhorn et al. 1983)

Antigenic profiles of in vitro-generated metacestode fractions were analyzed with respect to B- and T-cell epitope expression and with respect to Em2 antigen synthesis. Immunoblotting revealed the presence of antibodies directed against epitopes of vesicle fluids and metacestode extracts of in vitro-cultivated metacestodes in sera of patients suffering from E. multilocularis and E. granulosus infections. However, there were two major points of interest: First, the banding pattern of both vesicle fluids and metacestode extracts changed considerably when metacestodes were cultivated in the presence or absence of FCS. This indicated a differential expression of metacestode antigens according to culture conditions. Second, the vesicle fluids of in vitro-generated metacestodes showed a strong reaction with the $E$. granulosus-infected patient's serum (with a banding pattern very similar to that of the E. multilocularis-infected patient's serum), although the cellular metacestode vesicle walls did not. This indicated that although the vesicle-fluid components of both Echinococcus species are probably very similar in terms of antigenicity, the composition of their cellular antigens, including those situated on the acellular laminated layer, must be very different.

Lymphocyte proliferation assays have been a widely used tool to determine the presence of T-cell epitopes (Gottstein et al. 1994). We obtained blood from a human $\mathrm{AE}$ patient, and vesicle fluids and metacestode extracts of parasites cultivated in the presence and absence of FCS or in the presence and absence of feeder-cells as well as the respective medium supernatants were tested for lymphoproliferative potential. All in vitro-generated fractions induced proliferation of lymphocytes under the conditions used for this study. This demonstrated (1) that in vitro-generated $E$. multilocularis metacestodes carried epitopes recognized by T-cells from an E. multilocularisinfected patient and (2) that such T-cell epitopes were shed into the medium during in vitro cultivation. Due to the recently observed immune-suppression phenomena in murine $\mathrm{AE}$ with regard to susceptible C57BL/6 and AKR mice, it will be of special interest to search culture supernatants for parasite molecules responsible for this effect.

The presence of the Em2 antigen (Gottstein 1985) in various fractions of in vitro-cultivated metacestodes was demonstrated by double sandwich ELISA (Deplazes and Gottstein 1991). We found that the Em2 concentration in all fractions (vesicle fluids, metacestode extracts, and medium supernatants) was increased when the parasites 
were metabolically more active (e.g., in the presence of FCS and CACO2 feeder-cells) than when they were metabolically inhibited (e.g., in the absence of FCS). In addition, the presence of Em2 antigen in medium supernatnats confirmed the previous findings that antigens were released into the medium during in vitro cultivation.

It has previously been shown that the Em2 antigen plays an important role during initial parasite establishment within the host (Gottstein et al. 1994). This was also reflected by the surface accessibility of this antigen (Deplazes and Gottstein 1991). In addition, only those $E$. multilocularis metacestode structures that were positive for the Em2 antigen were capable of inducing secondary $\mathrm{AE}$ in rodents (Gottstein et al. 1992). Our results indicated that in vitro-cultivated, Em2-positive metacestodes also exhibited pathogenicity with respect to murine $\mathrm{AE}$. In in vitro-cultivated metacestodes the importance of this antigen in the initial host-parasite relationship was, as in vivo, reflected by its localization on the surface of the vesicles (as shown by direct immunofluorescence), and by the observation that the Em2 antigen was found, on sections, to be present exclusively on the outer layer of the metacestodes. No Em2 antigen was detected on internal metacestode structures such as protoscolices or the germinal layer. Electron microscope investigations finally showed the antigen to be a distinct component of the laminated layer. Dense labeling around the region where the microtriches protrude into the laminated layer suggests that Em2 is synthesized by tegumental tissue and is subsequently released into the laminated layer via the microtriches. It is not known whether the Em2 antigen is just transported through the laminated layer and released into its surroundings or whether this antigen actually plays an important structural role within the laminated layer itself.

Our findings suggest conclusively that in vitro-cultivated E. multilocularis larvae that are maintained and induced to proliferate in vitro under defined conditions show strong similarities to metacestodes generated in animals. These parasites are directly accessible for biochemical treatments and/or analysis without the caveat of unspecific host influence, and in vitro cultivation is therefore a valuable alternative to the animal experimentation that has been practiced to date. For many experiments it will be a major advantage to obtain pure metacestodes, especially in dissecting molecular mechanisms during differentiation and development of the parasite, as well as for studies on parasite interactions with defined host immune components.

Acknowledgements We are grateful to Rudolf Giovanoli (EM Laboratory, Institute for Chemistry, University of Bern) for allowing us access to his SEM facilities and to Toni Wyler (Institute for Zoology, University of Bern) for his hospitality at the TEM. We also acknowledge Norbert Mueller and Richard Felleisen (Institute for Parasitology, University of Bern) for their constructive criticism and critical reading of this manuscript. This work was supported by NF grant 31-29651.90.

\section{References}

Aggarwal A, Merrit JW, Nash TE (1989) Cysteine-rich variant surface proteins of Giardia lamblia. Mol Biochem Parasitol 32: $39-48$

Alkarmi TO, Alshakarchi Z, Behbehani K (1988) Echinococcus multilocularis: the non-specific binding of different species of immunoglobulins to alveolar hydatid cysts grown in vivo and in vitro. Parasite Immunol 10:443-457

Dao L (1985) An improved method of antigen detection on nitrocellulose: in situ staining of alkaline phosphatase conjugated antibody. J Immunol Methods 82: 225-231

Delabre I, Gabrion C, Contant F, Petavy AF, Deblock S (1987) The susceptibility of the mongolian gerbil (Meriones unguiculatus) and the OF1 mouse strain to Echinococcus multilocularis - ultrastructural aspects of the cysts. Int J Parasitol 17: 773-780

Deplazes P, Gottstein B (1991) A monoclonal antibody against Echinococcus multilocularis Em2 antigen. Parasitology 103: 41-49

Eckert J, Thompson RCA, Mehlhorn H (1983) Proliferation and metastases formation of larval Echinococcus multilocularis. I. Animal model and histological findings. Z Parasitenkd 69: 737-748

Gottstein B (1985) Purification and characterization of a specific antigen from Echinococcus multilocularis. Parasite Immunol 7: $201-212$

Gottstein B, Tsang VCW, Schantz PM (1986) Demonstration of species-specific and cross-reactive components of Taenia solium metacestode antigens. Am J Trop Med Hyg 35: 308-313

Gottstein B, Deplazes P, Aubert M (1992) Echinococcus multilocularis: immunological study on the "Em2-positive" laminated layer during in vitro and in vivo post-oncospheral and larval development. Parasitol Res 78: 291-297

Gottstein B, Eckert J, Frey H (1983) Serological differentiation between Echinococcus granulosus and $E$. multilocularis infections in man. Z Parasitenkd 69:347-356

Gottstein B, Wunderlin E, Tanner I (1994) Echinococcus multilocularis: Parasite-specific humoral and cellular immune response subsets in mouse strains susceptible (AKR, C57BL/6J) or "resistant" (C57BL/10) to secondary alveolar echinococcosis. Clin Exp Immunol 96: 245-252

Hemphill A, Affolter M, Seebeck T (1992) A novel microtubule binding motif identified in a high molecular weight microtubule-associated protein from Trypanosoma brucei. J Cell Biol 117: 95-103

Kennedy JR, Williams RW, Gray JP (1989) Use of Peldri II (a fluorocarbon solid at room temperature) as an atternative to critical point drying for biological tissues. J Electron Microsc Tech 11: $117-125$

Mehlhorn H, Eckert J, Thompson RCA (1983) Proliferation and metastases formation of larval Echinococcus multilocularis. II. Ultrastructural investigations. Z Parasitenkd 69: 749-763

Nahhas SA, Gabrion C, Walbaum S, Petavy F (1991) In vivo cultivation of Echinococcus multilocularis protoscolices in micropore chambers. Int J Parasitol 21: 383-386

Rausch R, Jentoft VL (1957) Studies on the helminth fauna of Alaska. XXXI. Observations on the propagation of the larval Echinococcus multilocularis. Leuckart, 1863, in vitro. J Parasitol 43: 1-8

Sakamoto T, Sugimura M (1970) Studies on echinococcosis. XXIII. Electron microscopical observations on histogenesis of larval Echinococcus multilocularis. Jpn J Vet Res 18:131-144

Smith M, Croft S (1991) Embedding and thin-section preparation: In: Harris JR (ed) Electron microscopy in biology. A practical approach. IRL-Press Oxford, pp 17-37

Thompson RCA, Lymbery AJ (1988) The nature, extent and significance of variation within the genus Echinococcus. Adv Parasitol 27: 209-258

Yamashita J, Ohbayashi M, Sakamoto T, Orihara M (1962) Studies on echinococcosis. XIII. Observations on the vesicular development of the scolex of $E$. multilocularis in vitro. Jpn J Vet Res 10: 85-96 